\title{
THE LUMINESCENCE OF THE IODIDE OF MILLON'S BASE
}

BY HARRY B. WEISER

The iodide of Millon's base, commonly called Nessler's precipitate, is obtained when Nessler's solution, an alkaline solution of mercuric potassium iodide, is treated with ammonia. According to Rammelsburg ${ }^{1}$ and to Pesci ${ }^{2}$ the structural formula of the compound is $\mathrm{N} \cdot \mathrm{Hg}_{2} \mathrm{I} \cdot \mathrm{H}_{2} \mathrm{O}$, while Franklin ${ }^{3}$ formulates it HOHgNHHgI. Physically the iodide is a chocolate-colored powder with a reddish brown tinge. Watts' Dictionary states that when heated the compound begins to give off water at $128^{\circ}$; heated more highly out of contact with air, it melts to a dark brown liquid and then decomposes. Roscoe and Schorlemmer add that the decomposition is accompanied by the emission of light.

Since the majority of chemiluminescent reactions studied ${ }^{5}$ have been those of combination or oxidation and not the reverse reactions of dissociation or reduction, it seemed desirable to investigate further the decomposition of the iodide of Millon's base to see whether the reaction emits light of a characteristic color and to add to the data previously obtained on mercury flames.

A quantity of the iodide was prepared by precipitating alkaline potassium mercuric iodide with ammonia. The precipitate formed was transferred to a Büchner funnel, washed until the wash water was neutral and finally dried in an oven at $115^{\circ}$.

A portion of the salt was placed in a tube and sealed to one of the exit tubes of a mercury-filled gas burette supplied

${ }^{1}$ Jour. prakt. Chem., (2) 38, 508 (1888).

2 Zeit. anorg. Chem., 21, 36I (1899).

${ }^{3}$ Franklin: Jour. Am. Chem. Soc., 29, 35 (1907); cf. Hofmann and Marburg: Liebig's Ann., 305, 204 (I899).

${ }^{4}$ Roscoe and Schorlemmer: Treatise on Chemistry, 2, 692 (I907).

${ }^{5}$ Trautz: Zeit. phys. Chem., 53, I (1905); Bancroft and Weiser: Jour. Phys. Chem., I8, 213, 281, 762 (I914); 19, 3 10 (1915). 
with a two-way stopcock. After evacuating the tube containing the iodide, it was slowly heated and decomposition took place. Qualitative analysis of the gas evolved showed it to consist of nitrogen and ammonia. Some ammonium hydroxide was formed by the union of ammonia with water vapor which was also a product of the action. Mercuric iodide and metallic mercury condensed on the tube above the heated portion. Contrary to the statements found in the handbooks, the decomposition of the compound took place without previous melting. Numerous attempts were made to fuse the compound, even heating it in a sealed tube under pressure, but all were unsuccessful. It was frequently observed that when a quantity of the salt was slowly heated in a test-tube, that the mercuric iodide formed in the decomposition condensed on the tube above the heated salt and when the temperature was raised fused to a red-brown liquid. It seems probable that this may have been mistaken for fusion of the undecomposed iodide of Millon's base.

A portion of the salt was heated in a test-tube immersed in a paraffine bath and an evolution of ammonia was detected with moist litmus paper at a temperature of $160^{\circ}$. The velocity of decomposition increased slowly with rising temperature but the critical reaction velocity necessary for the production of light was not reached until above $35^{\circ}$. In this respect the decomposition of the iodide is similar to the thermal decomposition of a number of exothermic compounds, e. g., $\mathrm{HgO}$, which begins to give off oxygen very slowly at as low a temperature as $175^{\circ}$ but does not undergo rapid decomposition until heated to redness.

The light emitted by the reaction is particularly bright so that it may be readily seen even in daylight. It furthermore possesses a distinctly characteristic color that at first reminded me of the violet potassium flame. On comparing the two colors it was found that the luminescence produced by the decomposition of the iodide was a darker, more distinct violet unmixed with the red luminescence that modifies the

${ }^{1}$ Schoch: Am. Chem. Jour., 29, 3 Ig (1903). 
violet of the potassium flame. On first observation it was thought that the violet light might be due to the presence of potassium iodide adsorbed by the precipitated salt. Although spectroscopic examination failed to disclose any of the characteristic lines of the potassium flame, a further test was carried out with the iodide precipitated from an alkaline solution of mercuric sodium iodide thereby removing any possibility of the presence of potassium. The decomposition of the :odide so prepared produced the same characteristic luminescence.

The question naturally arose whether this luminescent reaction is exothermic or endothermic. It is a well-known fact that in the majority of cases the thermal decomposition of a dry compound is attended by the absorption of heat. In some cases notably in explosive reactions and in the decomposition by heat of potassium chlorate and potassium bromate the decomposition is accompanied by an evolution of heat. Even when thrown onto a hot plate considerably above the decomposition temperature of the iodide the rapid decomposition can no more be regarded as explosive in character than the rapid decomposition of potassium chlorate which evolves heat or the rapid decomposition of silver oxide or potassium iodate which absorbs heat. This led to further study to determine whether the thermal effect was similar to that of most decomposition reactions or whether the decomposition was accompanied by an evolution of heat like potassium chlorate.

In order that a reaction may be accurately studied thermochemically it must take place rapidly at ordinary temperatures and must proceed rapidly to the end. If a reaction takes place rapidly only at elevated temperatures, it enormously complicates the accurate determination of the thermal effect and if the thermal effect is not particularly marked it even renders the determination of the heat sign a difficult procedure.

Attention has already been called to the fact that the decomposition of the iodide of Millon's base does not proceed with any great rapidity until around $400^{\circ}$ or above the range 
of use of an ordinary mercurial thermometer. It was thought, however, that the slow decomposition at a lower temperature might be accompanied by sufficient thermal effect so that the heat sign could be measured with an accurate thermometer. To try this an electric oven was heated to approximately $230^{\circ}$. By keeping the oven out of the region of air currents and by surrounding the thermometers with an asbestos jacket, a fairly constant temperature could be maintained that was read on a thermometer graduated in tenths of a degree. The bulb of a Beckmann thermometer was placed concentrically in a thin-walled test-tube and to hold it in place was surrounded with powdered barium sulphate. The tube was supplied with a cork which fitted the opening in the top of the oven and served to hold it firmly in the heated air. After waiting for more than an hour for the temperature to become constant the Beckmann was read. The thermometer was then surrounded with the iodide and the procedure repeated. Repeated attempts gave unsatisfactory results. Because of the inability to maintain the temperature absolutely constant at so high a temperature with the apparatus at hand, the reading on the Beckmann fluctuated during a long period of time through a few tenths of a degree, showing no marked tendency in either direction.

Various devices were made and tried out to utilize the heat of an electrically heated wire in a water calorimeter for effecting the decomposition of the salt and measuring the comparative rise in temperature of the water when a given quantity of the current was passed, with and without the presence and accompanying decomposition of the salt. Because of the slight thermal effect of the reaction compared with the heat of the current and because of the difficulty in correcting for the quantity of heat necessary to raise the salt from room temperature to the temperature of rapid decomposition, the method was found to be impracticable.

It seemed likely that the best results could be obtained by bringing about rapid decomposition of the iodide in a confined space and noting the effect on a thermometer the bulb 
of which was suspended in this space. A description of the apparatus that was finally constructed for this purpose and the method of manipulating it in the specific case of the decomposition of the iodide are as follows: A Hoskins' crucible furnace was connected with the source of current through a slide wire rheostat and regulated so that 1.8 amperes passed through the furnace. The furnace was allowed to heat until its temperature had become approximately constant, after which a $500 \mathrm{cc}$ round bottom flask supplied with a two-hole stopper was set snugly over the circular opening in the furnace and was clamped firmly in place. In one hole of the stopper was placed a thermometer, graduated from $100^{\circ}$ to $220^{\circ}$ in tenths of a degree and in the other hole was placed a glass tube of $3 \mathrm{~mm}$ bore, on the lower end of which was blown a thin-walled bulb that contained the I-gram sample of the iodide. A glass rod of suitable length was placed in the bulb for the purpose of breaking the bulb and releasing the salt at the desired time. Over the end of the bulb tube was placed a short piece of narrow rubber tubing that fitted the glass rod snugly. A short slit was cut in the rubber tubing making a Bunsen valve. The bulb of the thermometer was placed $4 \mathrm{~cm}$ from the bottom of the flask and was held firmly in place by a burette clamp. At the outset of the experiment the bulb containing the iodide was placed at the same level. For protection from air currents the entire apparatus was surrounded by a jacket of asbestos with a glass window in front, through which could be observed the thermometer reading and the reaction within the flask. When the thermometer was placed in the position above described and when the current passing through the furnace was I. 8 amperes the thermometer stood around $180^{\circ}$. When a constant temperature was obtained the bulb of salt was lowered until it was about $0.5 \mathrm{~cm}$ from the bottom of the flask and the thermometer was read after waiting until temperature equilibrium was restored. By a gentle tap, a hole was punched in the bottom of the bulb releasing the salt. By this procedure the salt was first heated to a temperature not far from the point of rapid de- 
composition and the bottom of the flask was such a temperature that the heated salt dropping on it immediately started to decompose with the emission of light. In a score or more of experiments carried out to secure the best conditions for the complete, rapid decomposition of the salt, the thermometer dropped from 3 to 5 degrees in the few seconds during which the reaction proceeded.

For securing the best results certain precautions other than those mentioned should be observed: It is undesirable to have the temperature of the bottom of the flask too high since the decomposition at the point of contact with the flask will be so rapid that the evolved gases will puff a large portion of the salt from the heated region. This also tends to fan up around the thermometer bulb, the more highly heated air in the bottom of the flask. For the same reason it is likewise best not to have the iodide precipitated on the bottom of the flask all at once but instead to have it run from the broken bulb in a stream, requiring a few seconds for emptying it completely. This is readily accomplished by breaking the bulb and then gently tapping the top of the bulb tube.

The thermometer dropped rapidly during the decomposition. This was followed by a short period of constant temperature and then by a uniform rise. Since the walls of the flask were coated with mercury and mercuric iodide which acted as a heat insulator, the thermometer naturally rose somewhat higher than the point at which it stood before the breaking of the bulb.

A logical objection may be raised to the result of this experiment, namely, that the condition in the flask which brought about the fall of the thermometer was not caused by the negative thermal effect of the reaction but instead was due to the quantity of heat necessary to raise the temperature of the salt from a point slightly under the temperature of rapid decomposition up to the point of rapid decomposition. A part of the lowering was unquestionably due to the latter effect but it seemed scarcely likely that this was the important thing. Even if we grant that the heat necessary to raise the 
heated salt to the temperature of rapid decomposition was sufficient to cause an appreciable lowering of the thermometer, the subsequent rapid decomposition, if it evolved heat, would cause it to rise or at least would slow down the rate at which it dropped. Observation showed that exactly the opposite was true.

It was found impossible to find a suitable substance with which to run a "blank" test, obviously because there is no substance which, when treated as the iodide was, would behave in a similar way without chemical action and corresponding heat changes coming in. However, a test was made to determine the effect on the thermometer when barium sulphate, an infusible, non-volatile substance was substituted for the iodide in the experiment above described. The procedure followed was identical with that above given as the best conditions for decomposing the iodide. It was found that the thermometer fell very slowly about 0.6 of a degree and then began to rise. Since decomposition of the iodide takes place at a lower temperature than that of the bottom of the flask and since the specific heats of the iodide and barium sulphate can not be very widely different, it is evident from the above experiment that the fall of the thermometer during the decomposition of the iodide cannot be accounted for solely by the difference in temperature between the iodide before breaking of the bulb and that at which the salt undergoes rapid decomposition. Of particular importance should be mentioned the additional fact that even the relatively slight lowering with barium sulphate was gradual and occupied a much longer time than the much greater lowering during the decomposition of the iodide.

To test the apparatus, the decomposition of certain compounds was carried out, the heat sign of which was known. A mixture of potassium chlorate and manganese dioxide in the proportion of about 3 to I caused the thermometer to rise from the start. Like results were obtained with ammonium nitrate. With silver oxide which absorbs a small amount of heat on decomposition, a slow fall in temperature was noted 
as long as the relatively slow decomposition took place, after which it rose gradually. That this fall in temperature was not due altogether to the quantity of heat necessary to raise the residue of silver to the temperature of the bottom of the flask, the experiment was repeated with the residue of silver alone. The fall in temperature was appreciably less.

In the light of these experiments we are led to the conclusion that the decomposition of the iodide of Millon's base with emission of light is accompanied by a negative thermal effect. Attention should be called to the fact that the temperature necessary for securing sufficiently rapid decomposition to obtain light is above the vaporization temperature of the reaction products.

The result of this investigation is important as an illustration of the fact that the thermal effect of a luminescent reaction is not necessarily positive. The result is merely illustrative of what must be true in a number of cases, since a number of luminescent reactions are already known that are accompanied by an almost imperceptible temperature change and since it is probable that all reactions emit light providing the reaction velocity is sufficiently high. ${ }^{1}$ That this reaction is further important as an illustration of the feasibility of obtaining light is evident from the following quotation from Bancroft:" "To obtain cold light one must find a reaction which can be made to go rapidly, which absorbs heat, or evolves but a small amount of heat and which has a high conversion factor for light."

\section{Luminescent Reactions of Mercury}

Investigation of flame reactions in which the element mercury is involved, discloses the presence of at least three characteristic and more or less separate and distinct luminescences: orange, green and violet.

The orange luminescence: An orange luminescence is ob-

${ }^{1}$ Bancroft: Jour. Franklin Inst., 175, 129 (1913).

2 The Theory of Cold Light: Trans. Ill. Eng. Soc., 10, 289 (I915). 
tained when cathode rays act on a mercurous salt, ${ }^{1}$ when mercury is made anode in the electrolysis of a chloride, bromide or iodide solution ${ }^{2}$ and near the mercury surface where mercury is in excess, in the combustion of the element in chlorine or bromine. The latter is best observed when just the surface of the mercury is uniting with the halogen with the emission of light rather than when a large flame is investigated.

Since in all of the above reactions the chief product is mercurous salt the reaction which produces the orange luminescence is the reaction from mercury to mercurous ion or mercurous salt. Inasmuch as the orange luminescence is not so marked in the spark spectrum of the element in vacuo, it is probable that the orange light is due chiefly to the reaction,

$\mathrm{Hg} \cdot \longrightarrow$ Non-dissociated mercurous salt.

The green luminescence: A green or greenish yellow luminescence is obtained when cathode rays act on mercuric chloride or bromide, ${ }^{3}$ when mercury burns in an excess of chlorine and in the electrolysis of concentrated sulphuric acid with a mercury anode. ${ }^{4}$ Under these conditions the mercuric salt is formed chiefly but the reaction does not take place to the exclusion of or without the intermediate formation of the mercurous salt. Hence whatever the color of the light emitted during the formation of mercuric salt from mercury, the color will necessarily be modified to a greater or lesser extent by the orange luminescence. ${ }^{5}$ In all the experiments on direct union which result in the formation of the mercuric salt, a green luminescence is obtained that is modified by the orange to such an extent that the color is best described as greenish or as greenish yellow. When mercury is burned in bromine, the orange light near the mercury surface where

\footnotetext{
1 Wilkinson: Jour. Phys. Chem., I3, 69I (I909); Farnau: Ibid., I7, 637 (1913).

${ }^{2}$ Wilkinson: Loc. cit.; Bancroft and Weiser: Jour. Phys. Chem., I8, 762 (1914).

${ }^{3}$ Farnau: Loc. cit.

${ }^{4}$ Wilkinson: Ibid.

${ }^{5} \mathrm{Cf}$. Bancroft and Weiser: Jour. Phys. Chem., I8, 28I (I9I4).
} 
mercury vapor is in excess is so bright that the body of the flame where mercuric salt is formed chiefly, appears distinctly yellow to the unaided eye. The tendency is in the right direction and is exactly what we should expect under the circumstances.

It is probable that the light emitted during the electrolysis of concentrated sulphuric acid with a mercury anode is due in part at least to a silent discharge. ${ }^{1}$

The green luminescence unmixed with the orange cannot be secured, as might be expected, by burning mercurous chloride or bromide in an excess of the halogen since the heat necessary to cause a sufficiently rapid reaction to emit light causes the readily decomposed mercurous salt to dissociate into its elements. In consequence the color of the emitted light is the same as that obtained by burning mercury in the halogen.

Inasmuch as the green luminescence is marked in the spark spectrum of the metal even in vacuum, it is probable that the green luminescence is due chiefly to the reaction $\mathrm{Hg}^{*} \rightarrow \mathrm{Hg}^{*}$. What light if any is due to the reaction from mercuric ion to mercuric salt has not been determined.

The violet luminescence: Attention has been called to the remarkably distinct violet luminescence obtained during the decomposition of the iodide of Millon's base. Since metallic mercury is one of the decomposition products of the salt it seems probable that a part at least of the violet luminescence is produced by the reaction $\mathrm{Hg}^{*} \rightarrow \mathrm{Hg}$. This seems all the more probable in view of the fact that the violet luminescence is marked in the spark spectrum of mercury.

The results of this paper may be summarized as follows:

( $\mathrm{r}$ ) The iodide of Millon's base decomposes on heating with the formation of ammonia, mercuric iodide, nitrogen, water and mercury. Ammonia is evolved at as low a temperature as $160^{\circ}$ but the decomposition is not rapid until about $400^{\circ}$. The decomposition takes place without previous melt-

${ }^{1}$ Wilkinson: Loc. cit.; cf. W. von Bolton: Zeit. Elektrochemie, 9, 913 (1903). 
ing. Under the conditions of rapid decomposition, the reaction emits light and absorbs heat.

(2) The light emitted by the decomposition of the iodide of Millon's base is particularly bright and is of a distinct violet color.

(3) A summary of the luminescent reactions of mercury has been given.

(4) The reaction of mercury to mercurous salt gives an orange luminescence; of mercurous salt to mercuric salt a green luminescence; and of mercuric salt to mercury a violet luminescence. It is probable that the orange luminescence is due chiefly to the reaction $\mathrm{Hg}^{*} \rightarrow$ nondissociated mercurous salt, the green luminescence to the reaction $\mathrm{Hg}^{*} \rightarrow \mathrm{Hg}^{*}$ and the violet luminescence to the reaction $\mathrm{Hg}^{\cdot} \longrightarrow \mathrm{Hg}$.

Department of Chemistry

The Rice Institute

Houston, Texas 